\title{
A system designed to monitor in-situ the curing process of sprayed concrete
}

\author{
S. Aparicio Secanellas ${ }^{1, *}$, M. G. Hernández ${ }^{1}$, I. Segura ${ }^{2,3}$, M. Morata ${ }^{4}$, J. J. Anaya ${ }^{1}$ \\ ${ }^{1}$ Instituto de Tecnologías Físicas y de la Información "Leonardo Torres Quevedo”, ITEFI (CSIC), 28006, \\ Madrid, Spain \\ ${ }^{2}$ Department of Civil and Environmental Engineering, Universitat Politècnica de Catalunya, Barcelona Tech, \\ C1, Barcelona 08034, Spain \\ ${ }^{3}$ Smart Engineering Ltd, C/Jordi Girona 1-3, Parc UPC - K2M, Barcelona 08034, Spain \\ ${ }^{4}$ COMSA, Barcelona, Spain \\ E-Mails: sofia.aparicio@csic.es; m.g.hernandez@csic.es; ignacio.segura@upc.edu; \\ miquel.morata@comsa.com; jj.anaya@csic.es
}

\begin{abstract}
In this paper a system is presented to monitor in-situ the curing process of sprayed concrete. This system consists of an instrumented trough based on wireless sensor networks for monitoring temperature/relative humidity and ultrasounds. The trough has been designed using a trapezoidal cast with several encapsulated transducers and an electronic system for generation, acquisition and processing of ultrasonic signals. A methodology for the measurement of ultrasonic velocity has also been proposed. This system has been proven to be a very good solution for in-situ monitoring of the curing process of sprayed concrete, being able to detect problems or defects in the material fabrication.
\end{abstract}

Keywords: sprayed concrete; in-situ monitoring; curing process; ultrasounds

\section{Introduction}

Currently sprayed concrete is used both for the execution of new infrastructures and for their repair. The rapidity in its placement and the obtaining of resistant properties in a short time makes this solution very useful for many applications. The main problems generated by the use of sprayed concrete are the rejections that occur when projecting the material. On many occasions the concrete is not well adhered to the surface on which it is being sprayed and it can become detached. This can be dangerous if you are concreting areas such as the vaults of tunnels or roof structures. The properties of the concrete mix with additives and the behaviour once sprayed depend on the ambient temperature, the humidity, the presence of water on the surface, how arid 
the aggregates are, the type of cement used, among others. Sometimes it is difficult to correctly combine all the variables since there is no absolute truth about how to act and make decisions for all the casuistry that can occur in a work $[1,2]$.

The first use of a methodology for curing control was based on monitoring the internal temperature of cementitious materials. The behaviour of the temperature inside the material reflects when hydration reactions occur during setting. The curing degree of the concrete material can be estimated from the product of its internal temperature and the curing time or from the hydration rate of cement. The concept of maturity assumes that the strength gain in cementitious materials is a combined effect of temperature and curing time. Different maturity functions exist in the literature, including the Maturity method based on the product of time and temperature [3,4], or the Knudsen model based on the product of time and hydration rate of cement [5]. The maturity index allows assessing the compressive strength of concrete in-situ [6]. A methodology for the quality control of sprayed concrete based upon the maturity method was proposed in [7].

Other methods employed to study the curing process of cementitious materials, are based on non-destructive techniques such as acoustic emission [8], electrical conductivity [9], radar [10], ultrasounds [11, 12]. The ultrasonic-based methods are suitable for monitoring the curing of materials cementitious since the propagation of the mechanical waves in the material studied can provide us with a good knowledge of the environment investigated. Ultrasound techniques are one of the most widely non-destructive techniques used. Parameters such as ultrasonic velocity [13], attenuation [14], reflection coefficient [15], or energy transmitted [16] are closely related to the mechanical properties of the material, in particular during the setting and hardening processes. If these parameters are monitored throughout the process can provide very useful information for understanding how hydration evolves [13, 17].

A methodology for the quality control of the curing process in precast concrete plants by non-destructive testing techniques was presented in [18]. This methodology allows the estimation of the strength gain of the precast elements during the curing process using two different procedures, one based on temperature and the other one on ultrasonic velocity measurements. In this paper, the joint use of models based on temperature and ultrasonic velocity allowed to take into account the influence of curing temperature, concrete dosage, type of aggregates or water/cement ratio among other factors. 
In [19] the influence of temperature on the correlation curves between the compressive strength and the ultrasonic velocity was studied for several types of concrete. They concluded that at early ages is important to take in consideration the curing temperature when correlation curves between UPV and compressive strength are established.

At present, the control of the acquisition of resistances of the shotcrete is done in troughs according to UNEEN 14487-2:2008 [20], which are moulds that are filled with the same concrete that is being sprayed on the site. According to the European standard UNE-EN 14488-2:2007 [21], two methods are available to evaluate the development of the compressive strength of sprayed concrete at early ages: the penetration needle test and the stud driving method $[22,23]$. These methods provide an indirect estimate of mechanical strength, from tabulated abacuses. These tests, apart from not providing direct data of resistance, present a large dispersion of results, which is a source of errors and increases the material consumption at work.

To overcome these disadvantages, in this paper, a system has been designed to monitor the curing process of sprayed concrete in-situ. This system consists of an Instrumented Trough (IT) based on wireless sensor networks for monitoring temperature/relative humidity $(\mathrm{T} / \mathrm{RH})$ and ultrasounds. The IT has been designed using a trapezoidal cast, called trough, with several encapsulated transducers, an electronic system for generation and acquisition of the signals and a processing of the acquired signals. In this paper a description of the monitoring system is presented as well as the methodology for the measurement of ultrasonic velocity in the IT. The system was used to monitor sprayed concrete in-situ during some phases of the construction of the tunnel of the B-40 in Viladecavalls (Barcelona, Spain). This system has been proven to be a very good solution for in-situ monitoring of the curing process of sprayed concrete being able to detect problems or defects in the material fabrication.

\section{Description of the measured system proposed}

The monitoring system proposed for sprayed concrete consists of an Instrumented Trough. This system is composed of a trapezoidal cast, called trough, with several encapsulated ultrasonic transducers, temperature and humidity sensors and an electronic system based on wireless communication networks that allow collecting the signals coming from the ultrasonic, temperature and humidity sensors during the setting and hardening processes. The IT is a trough used in the characterization tests of sprayed concrete. Sensors and the 
associated electronics have been designed in such a way that they are resistant both to the projection and to the environmental conditions where this projection is performed (tunnels, laboratory, etc.). The IT incorporates ultrasonic transducers and $\mathrm{T} / \mathrm{RH}$ sensors because in previous works have been demonstrated that using a calibration procedure, the compressive strength can be estimated from the temperature and ultrasonic velocity measurements [18].

Once the signals and measured data are collected in a conventional computer, they can be processed and the information from the signals to characterize the sprayed material can be extracted. Currently, this evaluation is done when the experiment is finished but it could be done "online" using this IT with different methodologies that are being carried out.

The IT system therefore consists of a trough, temperature, relative humidity and ultrasonic sensors, an electronic system for generation and acquisition of the signals and a processing of the acquired signals.

\subsection{Trough}

In this work, a standard metal trough was mechanized to incorporate the new ultrasonic transducer designed for the characterization tests in sprayed concrete. The mechanization of the trough was carried out with different objectives. On the one hand, to avoid sonic continuity between transducers, this allows that the greatest ultrasonic energy travels through the concrete. Another aspect to take into account in the mechanized of the trough was the decoupling that can occur due to the inherent shrinkage in the setting process. On the other hand, the materials used in the trough must resist the spray process and absorb the impact energy, mainly from aggregates. The system has to be watertight, to withstand the environmental conditions where the projection is made. It is also necessary that it be energetically self-sufficient for the duration of the monitoring to facilitate its use within the operations of the sprayed concrete.

For the ultrasonic transmission, different transducer types and configurations were proved: transducers attached to the material or to the trough at different positions. In both configurations two transducers operating in transmission mode were used. The transducers were directly placed on top of the material projected; Figure 1 option A. This configuration did not allow monitoring the setting process of the material since an ultrasonic coupling was not obtained once the material was projected due to its roughness and hardness. Later it was tried to stick them on the side faces of the trough, Figure 1 option B, but they were decoupled when the first 
retractions were produced. In this case, an ultrasonic transmission (UT) was also produced by the trough itself that masked the ultrasonic transmission in the sprayed material, Figure 2 option B.
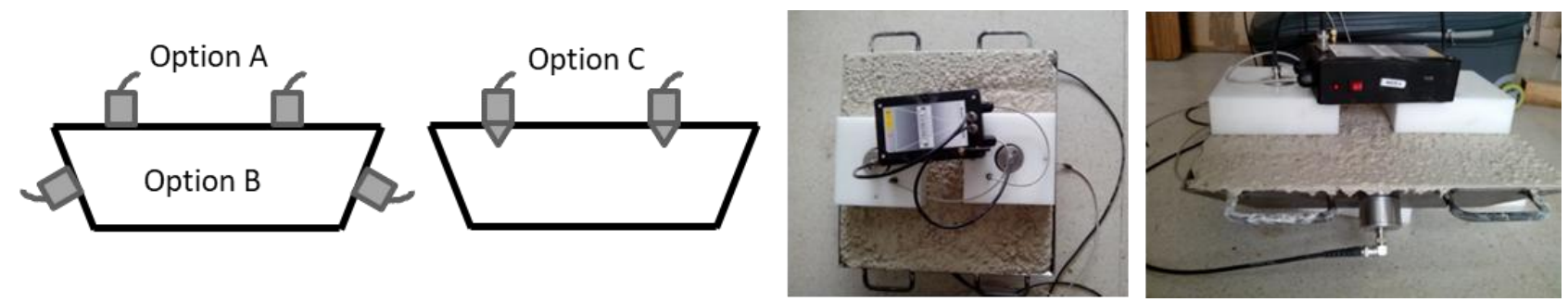

Figure 1. Different transducer configurations in the trough.

Another configuration probed was to glue the transducer with a metallic cone and placed this in the upper part of the trough once the material has been projected, Figure 1, option C. This configuration showed good results, as can be seen in Figure 2 Option C, but not is suitable for on-site use.
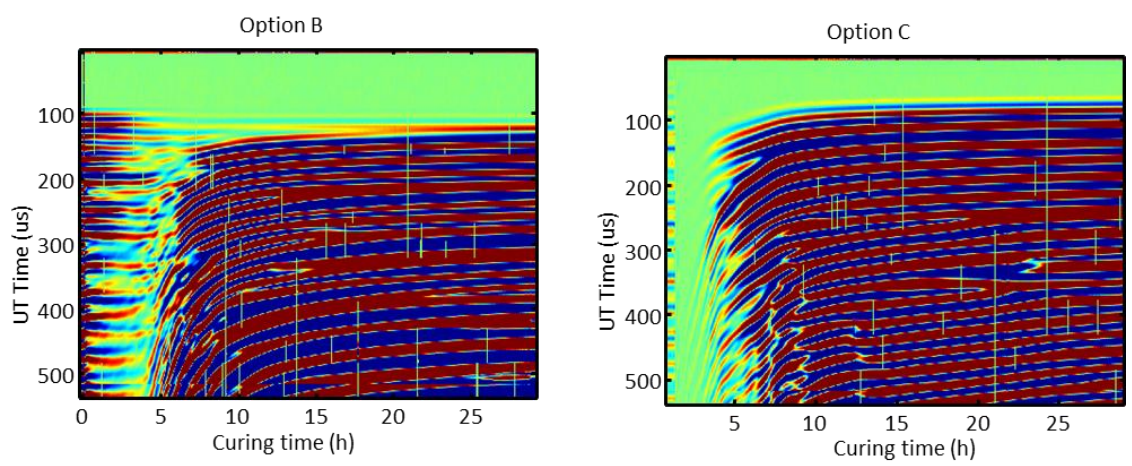

Figure 2. B-scan of monitorization of two troughs.

Therefore, impact-resistant stainless steel covers have been designed and manufactured and have been placed at the base of the trough. Two types of geometry, conical (C) and oblique cylindrical trunk (OCT) were tested, which allowed to improve the acoustic field emitted in the parallel direction to the bottom of the trough, Figure 3. The behaviour of these configurations of transducers was simulated and compared with real signals, and it is shown in section 2.2.1.

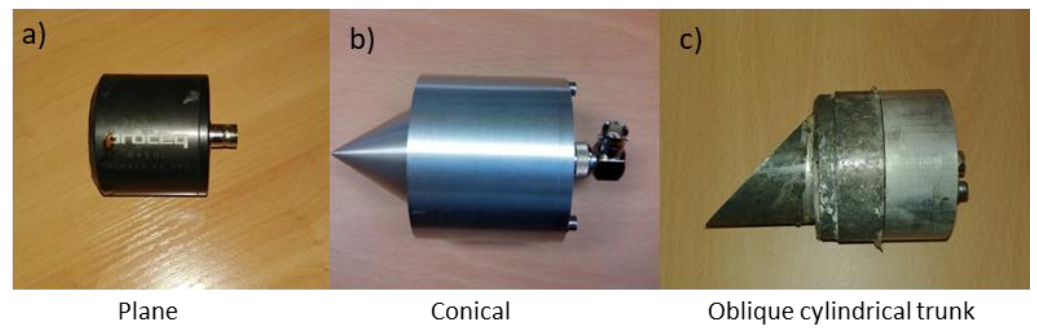

Figure 3. Transducers with different shapes. (a) Plane, (b) C, (c) OCT. 
The final solution adopted was to make 3 holes in the base of the trough for the ultrasonic transducers which endure the projection of concrete, Figure 4. The self-weight of the concrete in the trough prevents separation of material from the bottom of the cast and therefore, from the transducers. This way, when shrinkage occurs, ultrasonic coupling is maintained. The distance between the centres of the transducers has been determined to measure the ultrasonic velocity in two different zones and in two different distances. The short distance allows measuring the velocity as soon as possible after the projection is made. The long distance allows the ultrasonic pulse to cross more material, so the measurement of the ultrasonic velocity is more representative of the state and characteristics of the projected material. The transducers were separated from the trough and surrounded by a material resistant to projection, with high acoustic impedance, so that they are acoustically decoupled from the trough.

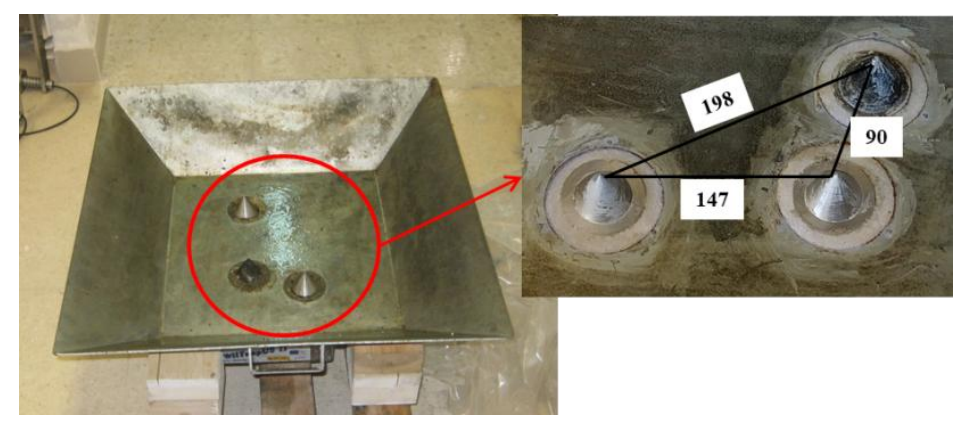

Figure 4. Position of the transducers in the trough.

\subsection{Sensors}

The system consists of temperature and humidity sensors and ultrasonic transducers. Environmental temperature and humidity sensors of SENSIRION series STH75 have been used. They have been conveniently encapsulated to protect them in the projection process. One of them has been arranged so that it measures the temperature of the trough and the humidity inside the electronic system. The other sensor measures environmental humidity and temperature.

For this application, ultrasonic transducers of $54 \mathrm{kHz}$ (of PROCEQ) were used inside stainless steel sleeves with conical geometry, Figure 3. The ultrasonic field generated by simulation and measurements in a water inspection tank was analysed in next section.

\subsubsection{Simulations of ultrasonic wave propagation in trough}


Numerical simulations of ultrasonic wave propagation in concrete were performed using COMSOL Multiphysics. A 2D model of acoustic modules was used for this study considering different geometry of ultrasonic transducers: plane, conical and oblique cylindrical trunk. The transducers size as well as their place into the trough is the same that in real experiments but only two transducers were considered in this simulation, a transmitter and a receptor.

The geometry of the model is shown in Figure 5. The trough has the same dimensions that in the real experiment. The geometry of the system was discretised using a finite element mesh. A free triangular mesh was used for all models with normal element size, Figure 5. The transducers are represented as a line placed at the bottom of the trough.
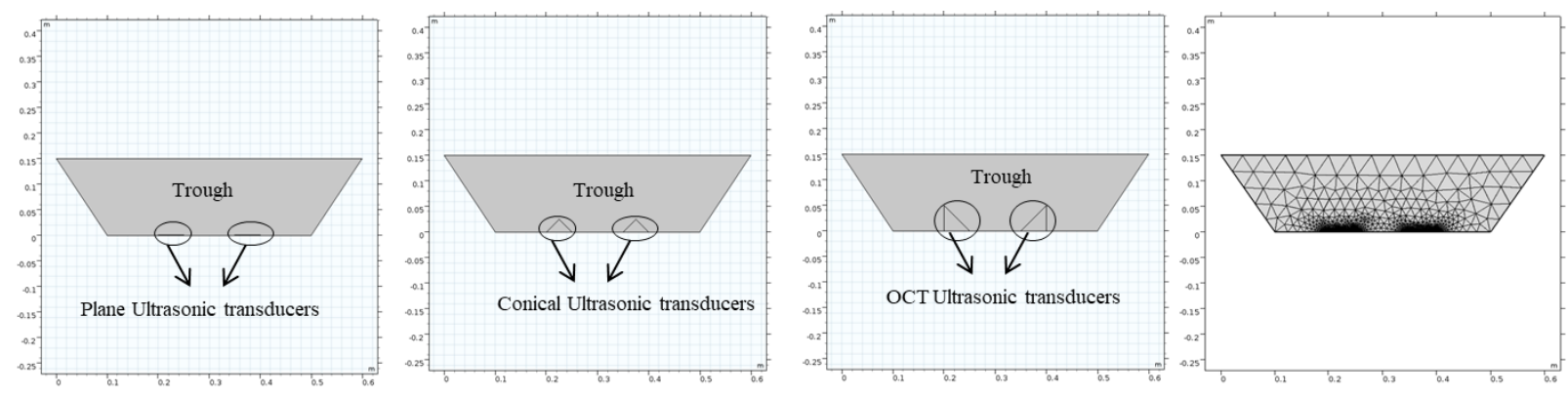

Figure 5. Geometry of the studied models and finite element mesh.

The acoustic wave, $p(t)$, was added to the system by applying a pressure wave to the transducers, Figure 5 . The applied pressure is defined as:

$$
p(t)=A \sin (t w) t^{2} e^{-3 B t}
$$

where $A$ is the amplitude, $w$ is angular frequency and $\mathrm{B}=20 \mathrm{kHz}$ corresponding to bandwidth pulse.

A time dependent solver was used in all simulations. The selection of the time step simulation is important for time dependent studies since the accuracy resolution and simulation duration can be controlled. The simulation time step should be less than ((wavelength/10)/ultrasonic velocity) to ensure mathematical stability [24]. The results considered in the simulations were the impulse transmission time and the amplitude of the received acoustic wave. The pressure received in the transducer was recorded at each time step and exported to MATLAB for post processing. The impulse transmission time was calculated by detecting the time at which the first part of the received pulse over an amplitude value of 0.1 was detected.

The ultrasonic field generated by the simulation in concrete was analysed, considering three types of geometry: plane, conical (C) and oblique cylindrical trunk (OCT). In Figure 6 the field generated by the three 
types of geometry is shown when the ultrasonic wave reaches the receiver transducer $(36.1 \mu \mathrm{s})$. The axes $\mathrm{x}$ and $y$ represent the sizes of the through and the false colour is the field of acoustic pressure transmitted in concrete. The conical and OCT geometries modify the wave propagation in comparison with the plane geometry, causing a lower energy transmission, a more dispersed field and a decrease in the wave amplitude. If we compare the conical and OCT geometries, we can see that the latter modifies the field creating a little more concentrated energy in the angular surface, which indicates that the ultrasonic field is redirected. In view of these behaviours we could conclude that the transducers with trunk-cylindrical shape present better characteristics.
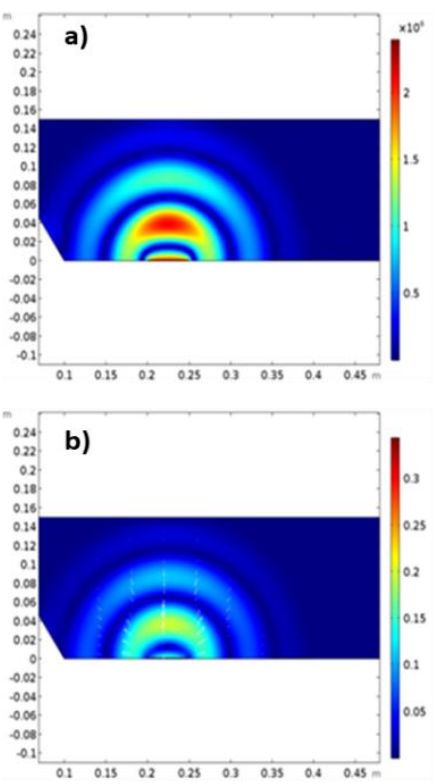

Plane
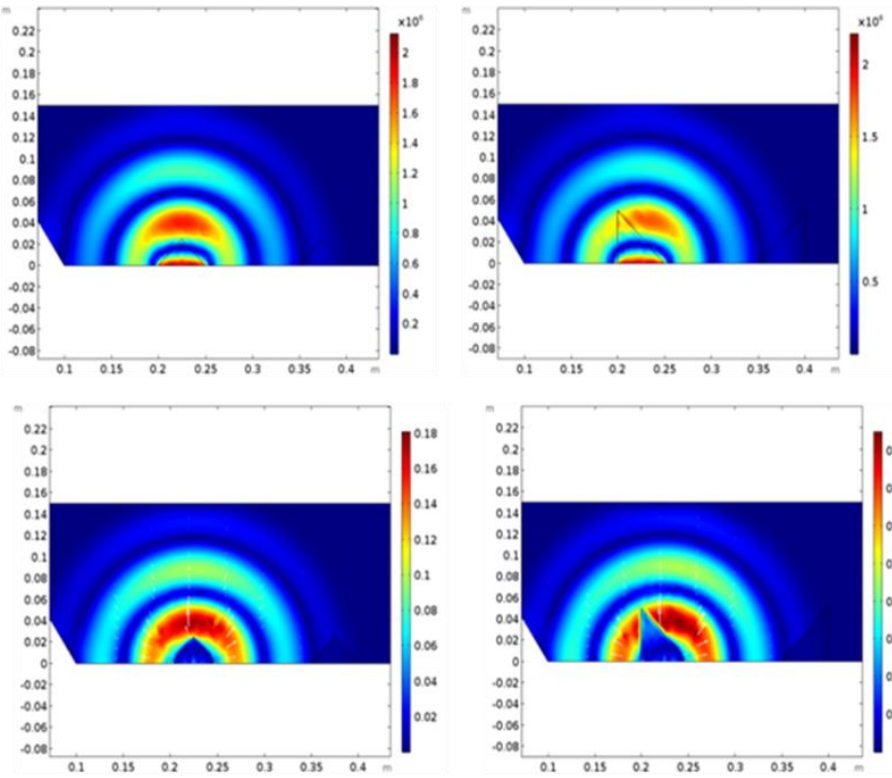

Conical

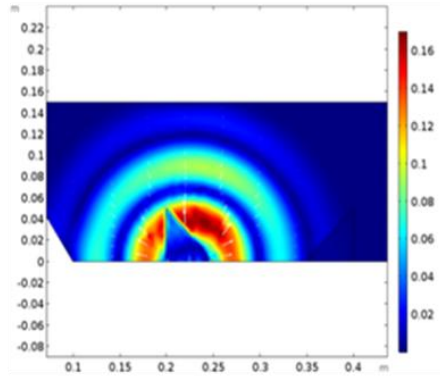

OCT

Figure 6. Ultrasonic field simulated with different transducers shapes. a) Pressure field, b) amplitude of acoustic velocity.

However, when the real tests were carried out using concrete and mortar, it was verified that all geometries behaved in a very similar way during the ultrasonic monitoring if we consider only the first semi-pulse, since the shape and amplitude of the signals acquired were very similar, see Figure 7. The first semi-pulse is the pulse usually considered to measure the ultrasonic velocity. The other semi-pulses are very different for the C and OCT shapes. This is due to the acoustic coupling in the OCT shape and to the lack of precision to simulate a conical shape with a 2D model in the C shape. Since the OCT shapes are more difficult to 
mechanize and to extract from the material once the monitoring has been carried out, the conical shapes were selected in the final system designed.
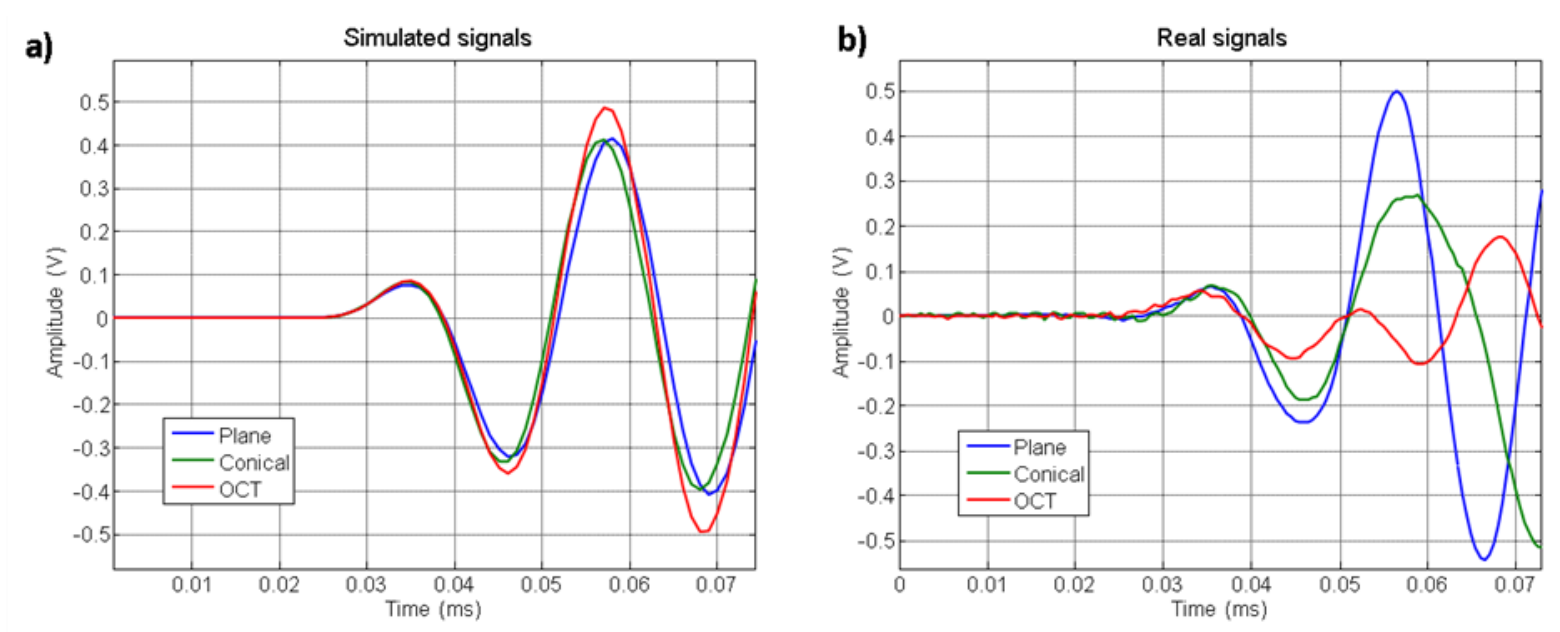

Figure 7. A-scan in concrete trough, a) simulation and b) real.

\subsection{System for generation and acquisition}

The system consists of a generating and acquiring ultrasonic signals equip and two sensors for measuring temperature and humidity (TH). This system was encapsulated in a watertight box resistant to the projection impact and to the weight of the concrete trough. This box was attached to the base of the trough, Figure 8 .

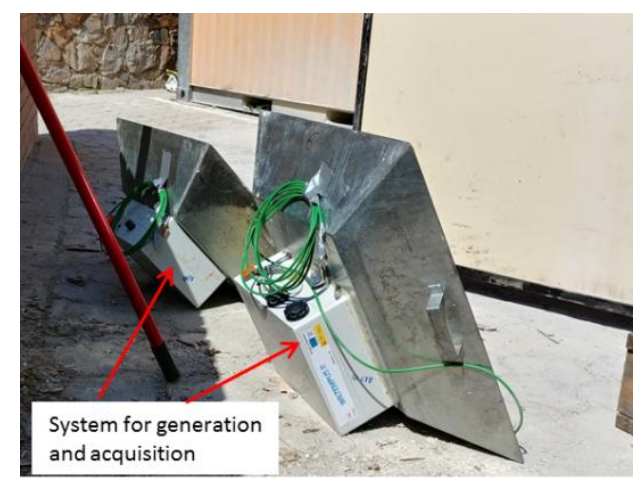

Figure 8. System for generation and acquisition attached to the trough.

The ultrasonic system was designed by the research group, based on an electronic board with an interface for wireless network motes from MEMSIC Company. In the experiments, IRIS model motes have been used. The system developed for the authors has 2 emission channels, which allows generating bipolar pulse trains of amplitude $\pm 8 \mathrm{~V}$; and 1 reception channel with a two-stage low noise amplifier with the following 
characteristics: 1st stage fixed gain of $20 \mathrm{~dB}$, 2nd stage programmable gain of $40 \mathrm{~dB}$ and bandwidth of 200 KHz. For all experiments, a sampling frequency of $1 \mathrm{MHz}$ has been used.

\subsection{Measurement system of temperature and humidity}

The two T/RH sensors used in this work are self-calibrated sensors and with I2C communication. The same board is used to receive the T/RH and the ultrasonic information. One sensor was attached to the trough to measure the temperature of the material projected thanks to the high thermal conductivity of the metallic trough. This sensor can also detect, by means of an anomalous increase in humidity, the possible leaks of water due to a lack of tightness, to an incorrect assembly or due to breakage of the box. Another sensor was used to monitor the environmental curing conditions studying the cement influence on the hydration reactions. This sensor is suitably protected from the aggressive environment of the projection process. For that purpose, an epoxy encapsulation and a GORETEX@ filter cap were used to prevent the ingress of dust and water, but allowing moisture to pass through. In Figure 9, and example of the T/RH measurements inside the IT and the ambient one are shown.

a)

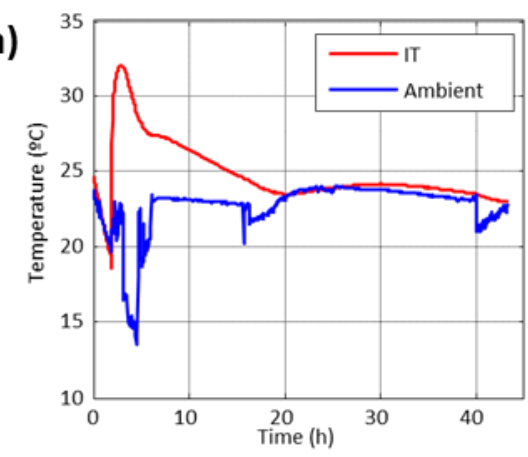

b)

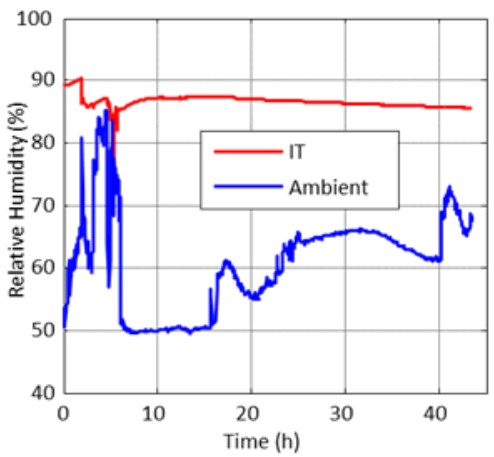

Figure 9. Example of behaviour of temperature a) and humidity b) in trough.

\subsection{Power supply system}

The power supply system consists of three rechargeable Ni-MH batteries of 4000mAH, which allows the system to operate at least 120 hours with a monitoring frequency of 1 acquisition per minute. The batteries are located in a watertight compartment accessible from the outside of the box, which facilitates their replacement. The charge level information of the batteries is also sent by the acquisition system so that the need to change the batteries can be detected. 


\section{Methodology for measurement of ultrasonic velocity}

The objective of the ultrasonic signal processing is to obtain the ultrasonic velocity during the process of setting and hardening of the sprayed material.

The messages content received from different motes must be analysed to eliminate the erroneous data and to concatenate the correct information for generating a data structure to process the ultrasonic signals. It must be considered that the environmental and operational conditions in civil works are harsh, with large metal machineries operating next to the monitored troughs, which causes interferences in the communications between the motes and the base station. This produces lost or erroneous messages that are removed to avoid generating false information. For each pair of transducers of each trough, a matrix containing the set of ultrasonic signals (BSCAN, Figure 10) and a vector with the information of the absolute acquisition time of each column of the matrix, which correspond to an ultrasonic signal (ASCAN, Figure 11) are generated. This allows the establishment of a relationship between the ultrasonic information and the elapsed time from the projection. In the BSCAN image of Figure 10, the removed messages by the analysis system can be clearly distinguished since they appear as vertical green lines.
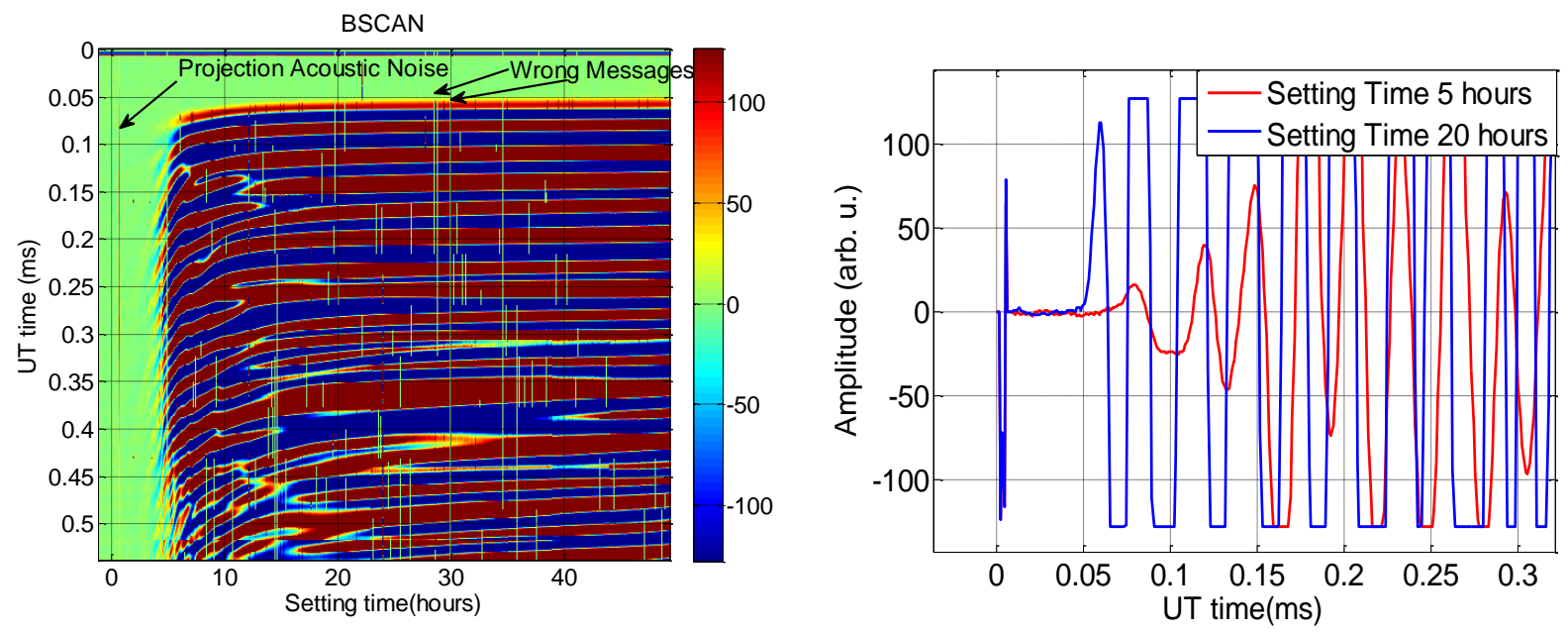

Figure 10. BSCAN.

Figure 11. Ultrasonic signals after 5 and 8 hours from the projection.

Processing is carried out to eliminate as much as possible the electronic and acoustic noise of the ultrasonic signals. The first unwanted electronic noise is the one induced by the excitation of the emitting transducer. This noise is easily eliminated just considering the signals from a certain time since this noise disappears after 
few microseconds from the transducer excitation. Since most of the signals are saturated, the frequency content of the signals varies as the curing takes place, and the temporal variation depends on the type of setting accelerator and the sprayed material, it is not convenient to use frequency band pass filters. Different algorithms were tested and it was determined that the signal noise could be reduced by using the digital envelope extraction by grinding and a digital low pass filter, Figure 12. There is no filter based on linear algorithms for decreasing acoustic noise, since they are generally impulsive noises and therefore their energy is in the entire frequency band including those of the ultrasonic transducers used. The solution adopted to reduce these impulsive acoustic noises is the use of a non-linear filter. The best results were obtained using a median filter of three values in the direction of hardening. This usual filter in image processing assigns to each pixel of the BSCAN (ultrasonic energy amplitude), the value of the median of three values formed by the immediately preceding and following pixel of the one considered:

$$
Y(n, m)=\operatorname{median}(X(n, m-1), X(n, m), X(n, m+1))
$$

where $n$ is the sample number of the ultrasonic signal, $m$ the number of ASCAN and $X$ the value of the ultrasonic amplitude or BSCAN and $Y$ the value of the filtered BSCAN.

This filtering has the advantage of, in addition to reducing the acoustic noise, allowing to eliminate any other value of the signal that does not have a correspondence in the ASCAN before or after the one that is analysed. For example, the number of removed messages because they are considered erroneous, is decreased, see Figure 13.

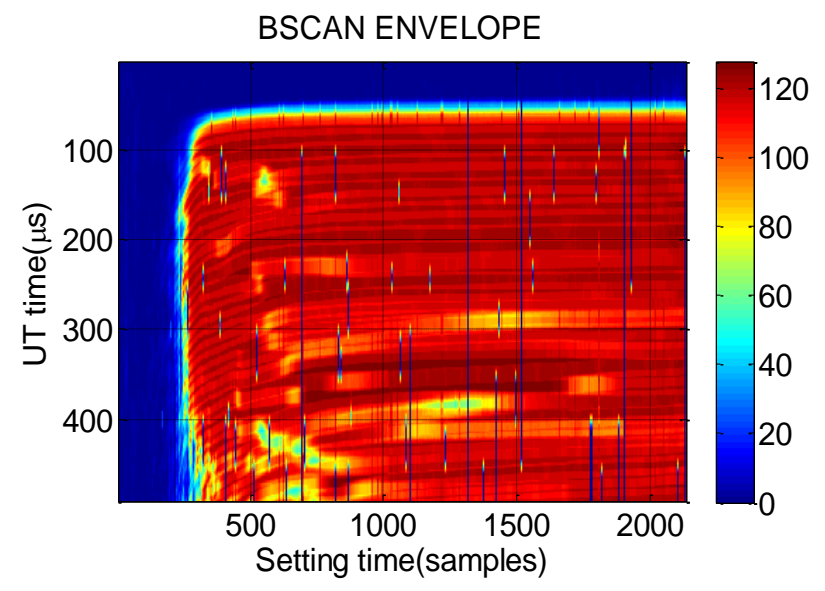

Figure 12. BSCAN of the envelope of the ultrasonic signals.

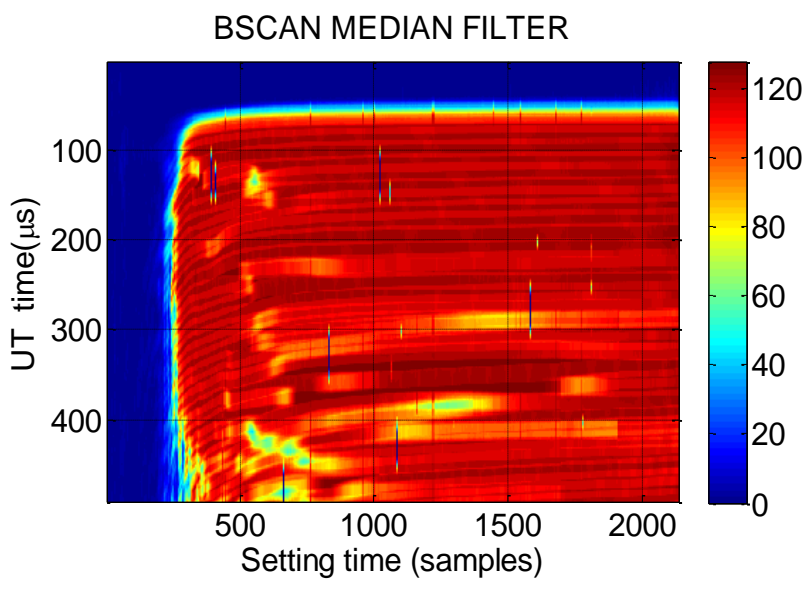

Figure 13. BSCAN after applying a median filter to the envelopes. 
There are three types of ultrasonic velocity, wave front velocity, phase velocity, and group velocity. In low dispersive materials at a certain frequency these three speeds coincide, but in the case of concrete during its curing phase these speeds are not equal. In addition, there is a problem associated when trying to measure the phase and group speed with low frequency transducers in structures, test tubes, witnesses or troughs of small dimensions. There are interferences between the emitted and reflected waves on the external surfaces. These interferences modify the phases and the position of the maximum value of the group speed, as can be seen in BSCAN of Figure 13, since if there were no interferences, the "scratching" would be parallel throughout the hardening time. For this reason, most measurements of the ultrasonic velocity in concrete use algorithms that detect the wave front. These algorithms are based on the detection of the initial flank of the ultrasonic pulse by exceeding an energy threshold. In short, the time from the generation of the electronic pulse in the emitting transducer is measured until the energy or amplitude of the suitably amplified ultrasonic signal exceeds a certain threshold value in the receiver transducer. The threshold is set by each device according to the sensitivity and electronic noise of the base station. In our case we used a fixed threshold of 3 applied to the logarithm value of the signal amplitude (Figure 14). In Figure 15 it can be seen that it is perfectly determined when the ultrasonic pulse is able to pass through the sprayed concrete between the two transducers.

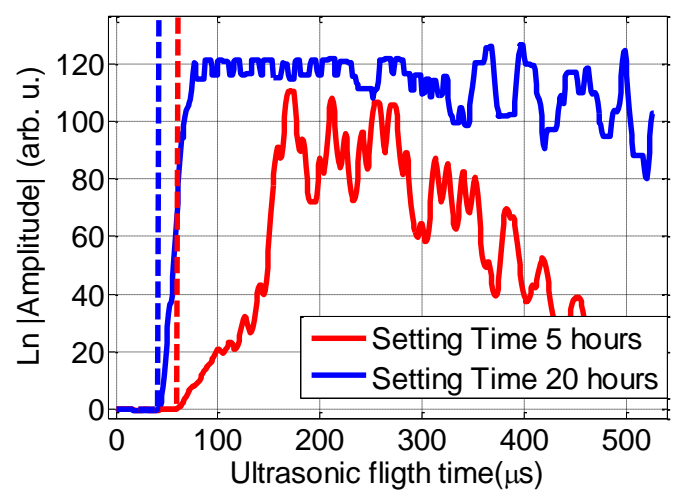

Figure 14. Pulse detection.

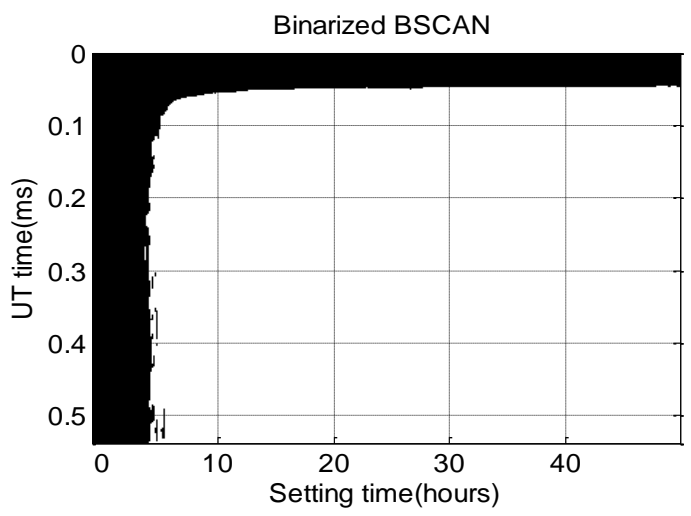

Figure 15. Result of the thresholding of the ultrasonic signals.

Once the crossing position by threshold $t(m)$ is determined the following formula can be used to compute the ultrasonic velocity:

$$
V(m)=\frac{d}{t(m)-e_{c}}
$$

Being $d$ the distance between the transducers, $e_{c}$ the absolute error of the time delay and $m$ the number of the ASCAN processed. The error $e_{c}$ has to encompass all the delays that are not due to the time that the ultrasonic 
pulse takes to pass through the material, for example the time it takes from the electronic excitation until the ultrasonic pulse begins to pass through the concrete. This delay is very difficult to calculate, it is constant for each trough and depends on the electronic system, transducers used, filters and processing applied, and can only be determined experimentally by means of a previous calibration of the troughs that will be discussed in the next section. On the other hand, another added difficulty is to determine the distance $d$ between transducers. It can be assumed that conical transducers located in the base of the troughs are used. Then, the distance between them corresponds to the distance between their centres. However, considering the simulation carried out and the calibration experiments, it was found that it is more convenient to use the distance between the bases of the cones. In the first phases of hardening, when the material is more attenuating, the energy that comes from this part of the transducers is not usually detected by the selected threshold. However when enough energy starts to pass that area produces the front of the detectable waves.

\subsection{System Calibration}

The calibration of a measurement system of ultrasonic velocity is usually a complex process that depends on of several factors as equipment, transducers, coupling, among others. The most used method consists on the determination of travelling time in standardized test piece or material, whose propagation time is known. The difference between these values is the measurement error, which is considered constant for each pair of transducers. In the proposed system, IT, different ways of calibrating the velocity measurement in troughs were probed using different types of coupling as water, methacrylate bars, aluminium. However, the obtained results cannot be extrapolated to materials as dispersive as mortar or concrete, due to the special geometry of the transducers and materials. Therefore, the following calibration methodology has been established:

1. Different mixes of mortar and concrete are carried out varying the dosage and the type of aggregate.

2. These mixes are poured into the trough, monitored for 48 hours and then the trough is unmoulded.

3. The velocity of the material is measured between the imprint of the transducers with a standard ultrasonic measurement system previously calibrated. For these measures, exponential conical transducers have been used that allow a point emission-reception. 
4. From these measurements, the measurement error parameter is determined for each pair of transducers used. Once this error is obtained, the velocity of the material projected during the hardening stage can be calculated, Figure 16.

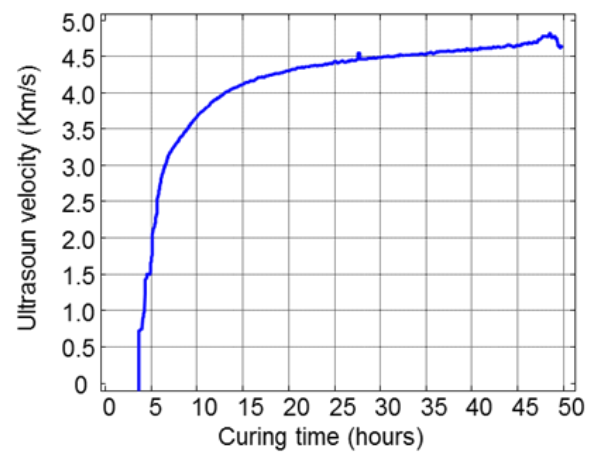

Figure 16. Ultrasonic velocity obtained using the signal processing method proposed.

\section{Results and Discussion}

The system presented in this paper was tested in-situ during the construction of the tunnel of the B-40 in Viladecavalls (Barcelona, Spain), see Figure 17. The results showed below correspond to a previous projection where different experiments were made using different dosages, accelerators and type of aggregates. During these projections, the aim was to determine the operative capacity of the monitoring system and its ability to detect problems in the setting process of the projected material. Therefore the failures that can occur in real projections were tested as for example, excess of water, rejection of aggregates, machines working.

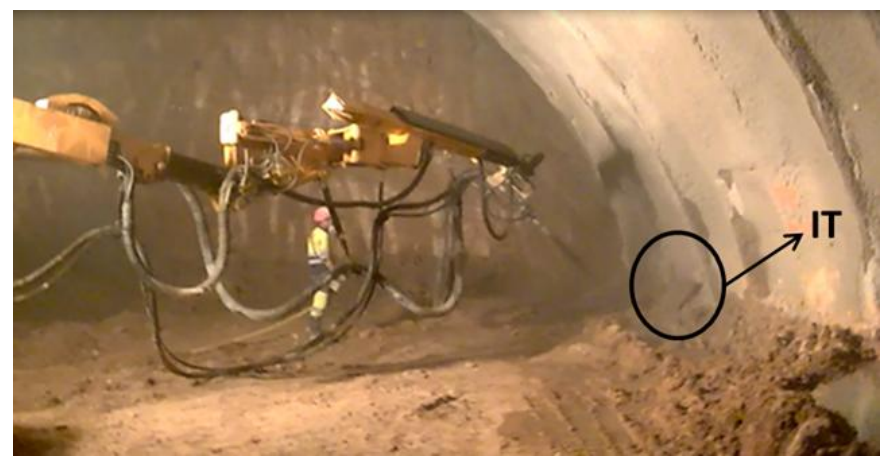

Figure 17. Concrete projection for the tunnel construction of the B-40 in Viladecavalls (Barcelona, Spain). For that purpose, 3 different ITs were tested during three different projections of recycled concrete to monitor the first 40 hours of curing, Figure 18a. In Figure 18b the positions of the transducer on each IT can be seen. 
a)

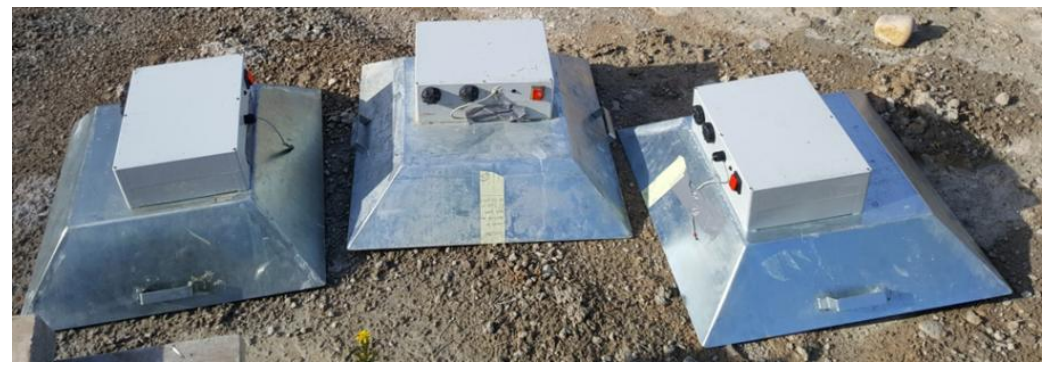

b)

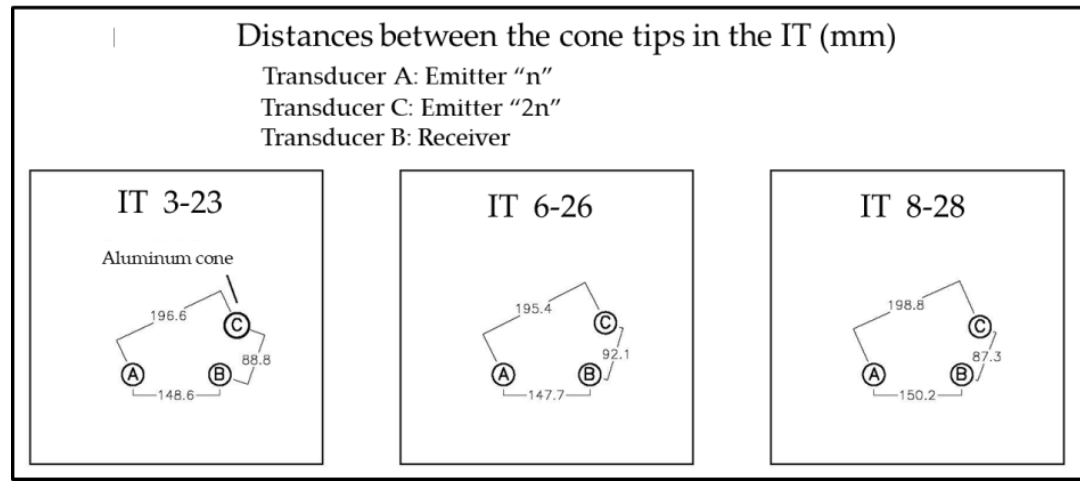

Figure 18. a) IT used in during projections in tunnel of the B-40 in Viladecavalls (Barcelona, Spain).

b) Description of the transducers positions on each IT.

In Figure 19 the temperature and relative humidity measured with each trough are shown. The setting and hardening processes can be seen during the first 40 hours.

a)
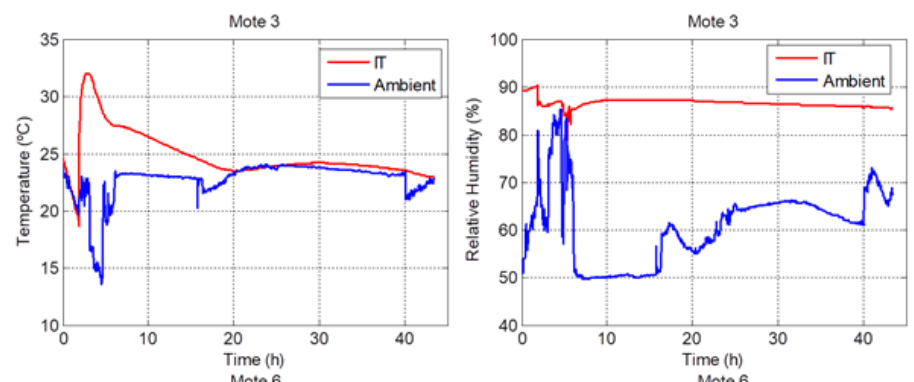

b)
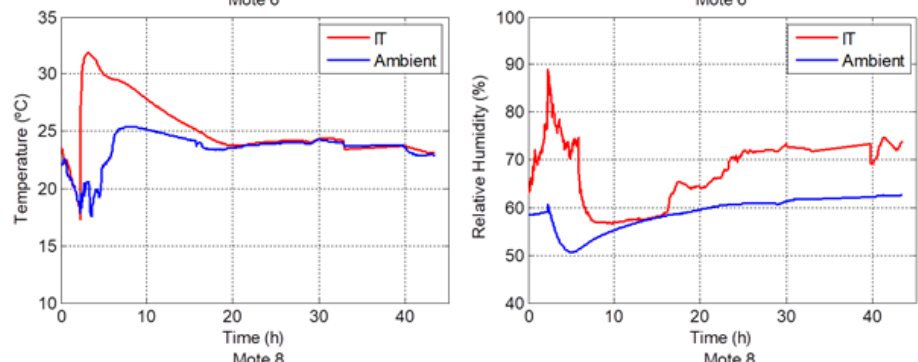

c)
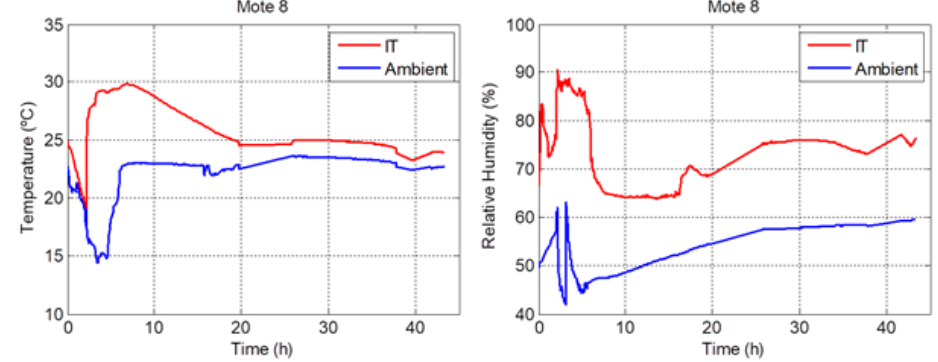
Figure 19. Temperature and relative humidity measured on each IT, a) 3, b) 6 and c) 8 .

In Figure 20 and 21 the ultrasonic results obtained on each IT using transducers " $n$ " (long distance) and " $2 n$ " (short distance) are shown. Observing the BSCANs of the acquired US signals, some aspects can be highlighted. With respect to the amplitude of the signals, it can be seen that signals are hardly transmitted in mote 8 and 28. It can also be seen that in mote 23 it has less signal than 3 when it should have greater amplitude since transducer 23 was closer than 3. With respect to the sonic noise, it can be noticed that motes 6 and 26 have moments of high noise from sample 600, due to proximity of the work machinery. In motes 8 and 28, the ultrasonic signal barely exceeds the ambient noise.

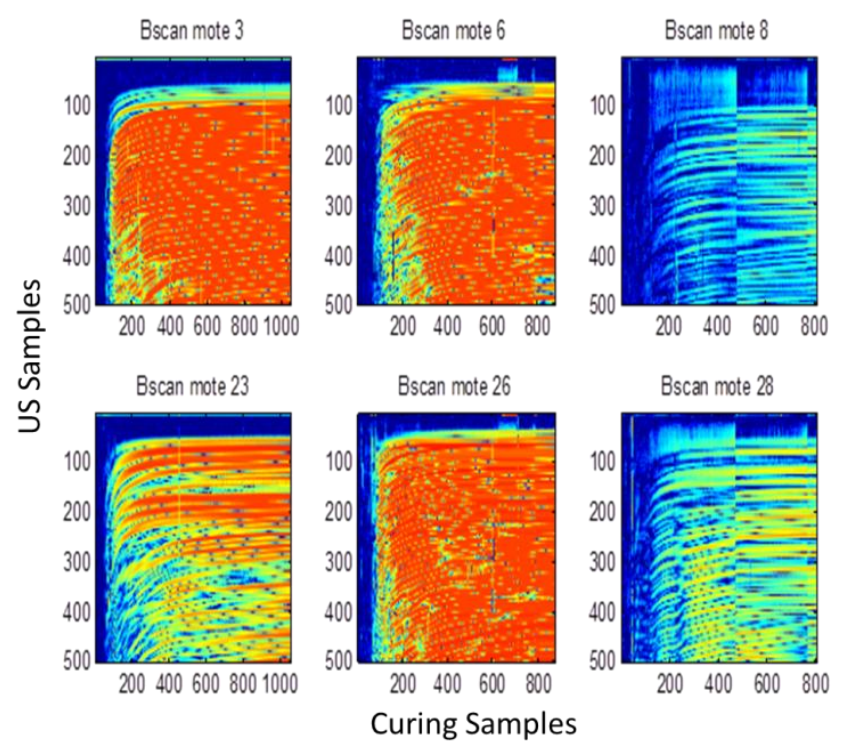

Figure 20. B-scan obtained on each trough using transducers "n" (long distance) and "2n" (short distance).

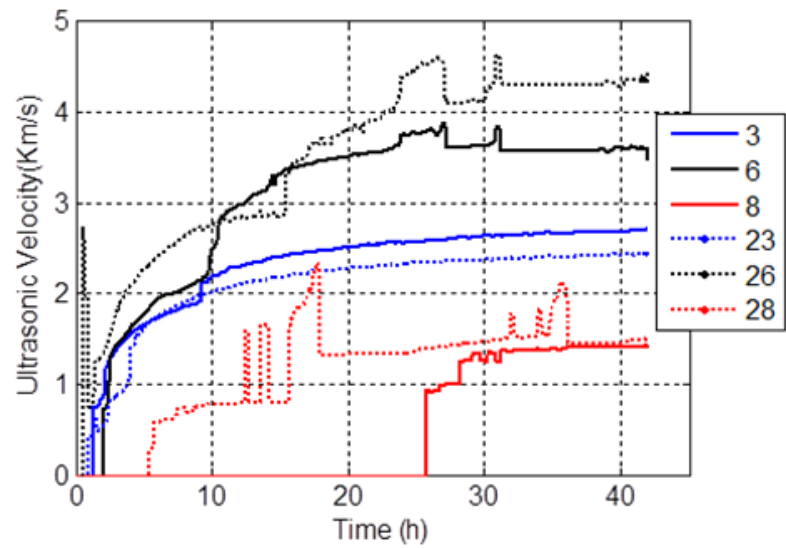

Figure 21. Ultrasonic velocity measured on each trough using transducers " $\mathrm{n}$ " (long distance) and " $2 \mathrm{n}$ " (short distance). 
Once the ultrasonic speed has been calculated, we can verify that although the sonic noise cannot be completely eliminated, important conclusions can be drawn from the quality of the sprayed material if a joint analysis with the monitoring temperature in the different troughs is made.

Although the temperature and RH results measured with Mote 3 show good results, the ultrasonic velocity was very low, because there was a lack of aggregates and therefore the material was not so good. Mote 6 presents good results but the noise can be seen in the ultrasonic measurements. While Motes 3 and 23 show similar velocity behaviour in Figure 21, there is a considerable gap in the velocities measured by Mote 6 and 26 after 30 hours of curing, which indicates that the projection was not uniform. Comparing the T/RH and US results measured with IT 8 , it can be seen that there was a problem with the material measured in this projection, because there was a water excess.

\section{Conclusions}

A sprayed concrete monitoring system based on the acquisition of ultrasonic signals, temperature and humidity has been shown in this paper. A methodology for the measurement of ultrasonic velocity has also been proposed.

The system was used to monitor sprayed concrete in-situ during some phases of the construction of the tunnel of the B-40 in Viladecavalls (Barcelona, Spain). It has been proven that, mechanically, the system designed by the authors, perfectly resists the projection environment being able to detect problems or defects in the manufacture of the material. Noise elimination processing was adequate to generate good velocity curves.

The monitoring of the ultrasonic signals easily detected the state of the curing of the projected material and the quality of the projected material. The non-uniform setting of the projected material was detected thanks to the measurement of the ultrasonic transmission velocity in two different trajectories. It was also found that using only temperature measurements, it is possible to detect an anomalous setting in the projected material but not a lack of aggregates. Instead this was detected with the ultrasonic velocity.

This system can also evaluate the compressive strength and the curing process by combining both the ultrasonic velocity and internal temperature data, in addition to humidity. Therefore, this system has been proven to be a very good solution for in-situ monitoring of the curing process of sprayed concrete, being able to detect problems or defects in the material fabrication. 


\section{Acknowledgments}

The authors will also like to thank COMSA and IQE companies for supplying the material of this work and for the technical support during the material projections.

Funding: This work was supported by Ministerio de Economía y Competitividad of Spain in the Call RetosColaboración 2015 [RTC-2015-3185-4 (MAPMIT)] and in the Call Retos Investigación [BIA2016-77992-R (AEI/FEDER, UE)]. Both projects were co-funded by the European Union through FEDER funds under the objective of promoting the technological development, innovation and high quality research.

\section{References}

[1] R. Dimmock, Sprayed concrete-Advanced technologies, Concrete, 37 (2003) 14-18.

[2] D. Zangerle, The use of wet mix sprayed concrete, in Tunnels and Metropolises, Negro Jr and Ferreira (eds), Taylor \& Francis, London, pp. 861-867, (1998).

[3] N. J. Carino, H. S. Lew, The maturity method: From theory to application. In Peter C. Chang, editor, Proceedings of the 2001 Structures Congress \& Exposition, ASCE, 2001, pp. 19.

[4] N. J. Carino, The maturity method: theory and application, Cem. Concr. Aggreg. 6 (1984), 61-73. https://doi.org/10.1520/CCA10358J.

[5] T. Knudsen, The dispersion model for hydration of portland cement I. General concepts, Cem. Concr. Res. 14 (1984), 622-630. https://doi.org/10.1016/0008-8846(84)90024-3

[6] C. Vázquez-Herrero, I. Martínez-Lage, F. Sánchez-Tembleque, A new procedure to ensure structural safety based on the maturity method and limit state theory, Constr. Build. Mater. 35 (2012), 393-398. https://doi.org/10.1016/j.conbuildmat.2012.04.040

[7] I. Galobardes, S. H. Cavalaro, C. I. Goodier, S. Austin, A. Rueda, Maturity method to predict the evolution of the properties of sprayed concrete, Constr. Build. Mater. 79 (2015), 357-369. https://doi.org/10.1016/j.conbuildmat.2014.12.038 
[8] K. Van Den Abeele, W. Desadeleer, G. De Schutter, Active and passive monitoring of the early hydration process in concrete using linear and nonlinear acoustics, Cem. Concr. Res. 39 (2009), 426 - 432. https://doi.org/10.1016/j.cemconres.2009.01.016

[9] J. M. Cruz, I. C. Fita, L. Soriano, J. Paya, M. V. Borrachero, The use of electrical impedance spectroscopy for monitoring the hydration products of Portland cement mortars with high percentage of pozzolans, Cem. Concr. Res. 50 (2013), 51-61. https://doi.org/10.1016/j.cemconres.2013.03.019

[10] C. Amer-Yahia, T. Majidzadeh, Inspection of Insulated Concrete Form walls with Ground Penetrating Radar. Constr. Build. Mater. 26 (2012), 448-458. https://doi.org/10.1016/j.conbuildmat.2011.06.044

[11] W. L. Lai, S. C. Kou, W. F. Tsang, C. S. Poon, Characterization of concrete properties from dielectric properties using ground penetrating radar, Cem. Concr. Res. 39 (2009), 687-695. https://doi.org/10.1016/j.cemconres.2009.05.004

[12] G. Trtnik, M. Gams, Recent advances of ultrasonic testing of cement based materials at early ages, Ultrasonics 54 (2014), 66-75. https://doi.org/10.1016/j.ultras.2013.07.010

[13] H. W. Reinhardt, C. U. Grosse, Continuous monitoring of setting and hardening of mortar and concrete, Constr. Build. Mater. 18 (2004), 145-154. https://doi.org/10.1016/j.conbuildmat.2003.10.002

[14] T. Voigt, T. Malonn, S. P. Surendra, Green and early age compressive strength of extruded cement mortar monitored with compression tests and ultrasonic techniques, Cem. Concr. Res. 36 (2006), 858-867. https://doi.org/10.1016/j.cemconres.2005.09.005

[15] G. Trtnik, M. Vali`c, F. Kav`ci`c, G. Turk, Comparison between two ultrasonic methods in their ability to monitor the setting process of cement pastes, Cem. Concr. Res. 39 (2009), 876-882. https://doi.org/10.1016/j.cemconres.2009.07.002

[16] N. Robeyst, C.U. Grosse, N. De Belie, Measuring the change in ultrasonic p-wave energy transmitted in fresh mortar with additives to monitor the setting, Cem. Concr. Res. 39 (2009), 868-875. https://doi.org/10.1016/j.cemconres.2009.06.016

[17] T. Voigt G. Ye, Z. Sun, S. P. Shah, K. van Breugel, Early age microstructure of Portland cement mortar investigated by ultrasonic shear waves and numerical simulation, Cem. Concr. Res. 35 (2005), 858-866. https://doi.org/10.1016/j.cemconres.2004.09.004 
[18] J. Ranz, S. Aparicio, J. V. Fuente, J. J. Anaya, M. G. Hernandez, Monitoring of the curing process in precast concrete slabs: An experimental study, Const. Build. Mater. 122 (2016), 406-416. https://doi.org/10.1016/j.conbuildmat.2016.06.041

[19] M. Velay-Lizancos, I. Martinez-Lage, M. Azenha, P. Vázquez-Burgo, Influence of temperature in the evolution of compressive strength and in its correlations with UPV in eco-concretes with recycled materials, Const. Build. Mater. 124 (2016), 276-286. https://doi.org/10.1016/j.conbuildmat.2016.07.104

[20] European Committee for Standarization, 'EN 14487-1:2008 'Sprayed concrete - Part 1: Definitions, specifications and conformity', 2008.

[21] European Committee for Standardization, EN 14488-2:2007 'Testing sprayed concrete - Part 2: Compressive strength of young sprayed concrete', 2007.

[22] J. Starlin Cedeño, Estudio de la Evolución de las Propiedades Mecánicas de Pastas y Morteros Proyectado Empleando Ultrasonidos, Tesis de Máster, UPC, 2015. http://hdl.handle.net/2117/78866

[23] S. Austin. Sprayed concrete technology. CRC Press, 2014.

[24] P. S. K. Mylavarapu, S. Boddapati, Numerical Modeling of Wave Propagation in Particulate Composites, Proceedings of the 2011 COMSOL Conference in Bangalore, 2011. 